Article

\title{
Influence of Organo-Sulfur Compounds with Overbased Calcium Compounds on Lubrication in Cold Forming
}

\author{
Tomohiro Takaki ${ }^{1, *}$, Kazuhiro Yagishita ${ }^{1}$, Teppei Tsujimoto ${ }^{1}$ and Toshiaki Wakabayashi ${ }^{2}$ \\ 1 Lubricants Research Laboratory, JX Nippon Oil \& Energy Corporation, 8 Chidori-cho Naka-ku, \\ Yokohama-shi, Kanagawa 231-0815, Japan; yagishita.kazuhiro@jxgr.com (K.Y.); \\ tsujimoto.teppei@jxgr.com (T.T.) \\ 2 Faculty of Engineering, Kagawa University, 2217-20 Hayashi-cho, Takamatsu, Kagawa 761-0396, Japan; \\ twaka@eng.kagawa-u.ac.jp \\ * Correspondence: takaki.tomohiro@jxgr.com; Tel.: +81-45-625-7151
}

Academic Editor: Maria Dolores Bermudez

Received: 29 December 2016; Accepted: 7 March 2017; Published: 16 March 2017

\begin{abstract}
The authors analyzed the structures of sulfurized olefins using NMR spectroscopy and studied the effects of sulfur chain length and alkyl structure on the ironing performance. They found that branched chain olefins, which contain branched alkyl groups, show superior ironing performance to straight chain olefins, provided that their carbon numbers are relatively low. When the sulfurized olefins were used in combination with overbased detergents (calcium sulfonate or salicylate), they showed a higher performance in ironing than with sulfurized olefins alone. It was also found that lubricating films consisting of both iron sulfide and calcium carbonate seem to improve ironing performance.
\end{abstract}

Keywords: organo-sulfur compound; sulfurized olefin; cold forming; ironing; overbased detergent; calcium sulfonate; calcium salicylate

\section{Introduction}

In metal forming processes such as ironing, the contact pressure between the tool and workpiece surfaces is extremely high and a great amount of heat is generated by friction and deformation [1]. Organo-sulfur and/or phosphorous compounds are widely used in metal forming fluids as extreme pressure (EP) additives due to their excellent performance in metal forming applications. Numerous studies on organo-sulfur compounds have been reported over the years. Allum et al. studied the relationship between the chain lengths of the alkyl groups in dialkyl disulfides and extreme-pressure performance [2]. Mori et al. reported that the thermal stability of EP additives plays a role in the amount of tribofilms that form on steel surfaces [3]. More recently, Mori et al. used a controlled atmosphere machining apparatus to elucidate the reaction mechanism of sulfurized olefins on nascent surfaces of transition metals [4]. In actual lubricating oil, various additives are used and, in particular, it is well-known that using organo-sulfur compounds in combination with overbased sulfonates produce better results in metal working than those provided by organo-sulfur compounds alone. Wakabayashi et al. analyzed chip surface after cutting and reported that when a polysulfide was used in combination with overbased calcium sulfonate, calcium carbonate promoted the formation of a lubricating film of iron sulfide [5]. Using Time of flight secondary ion mass spectrometry (TOF-SIMS), Kubo et al. analyzed the reaction films that formed when dibenzyl sulfide was used with overbased calcium sulfonate. They reported that iron sulfide forms on the substrate and that calcium oxide, the product of a tribochemical reaction involving calcium carbonate, forms on top of the 
iron sulfide [6,7]. Costello et al. used X-ray absorption near edge structure (XANES) analysis to study the effectiveness of using sulfurized olefins together with various metal sulfonates [8]. They found that overbased sulfonates accelerated the decomposition of sulfurized olefins, such that calcium sulfate formed on the topmost surface through an oxidation reaction and iron sulfide, rather than iron sulfate, formed in the bulk under the surface, thereby improving the extreme-pressure performance. Meanwhile, Sembongi et al. studied the effects of sulfur chain length and alkyl structure on cutting performance [9]. In addition to the aforementioned studies, many studies have been published over the decades concerning the lubricating properties of sulfur-based additives and their effectiveness when used together with overbased sulfonate. However despite this, little has been published on the detailed chemical structure of these sulfur compounds in connection with cold forming performance [10]. Moreover, almost nothing has been published that focuses on the molecular structure in cases where organo-sulfur compounds have been used in combination with overbased calcium compounds for cold forming. On the other hand, in their study of phosphorus compounds, Ohmori et al. conducted a ball penetration test which simulated cold forging to clarify the relationship between the alkyl chain length and anti-galling performance [11].

For this study, we prepared a number of sulfurized olefins with well-defined molecular structures and examined their performance by way of a burring test $[12,13]$, which can simulate the condition of cold ironing. It is thought that the combinatorial effect of using organo-sulfur compounds with overbased calcium sulfonate is closely related to the calcium carbonate contained within the micellar cores provided by the soap group of the sulfonate. We therefore carried out experiments to determine the influence of the soap groups on the performance of sulfurized olefins in combination not only with calcium sulfonate but also with calcium salicylate that has a different soap group structure. We also used X-ray photoelectron spectroscopy (XPS) and Auger electron spectroscopy (AES) analyses to study the tribofilms that formed on the metal contact surfaces during the ironing process and considered the composition of these lubricating films.

\section{Experimental Methods}

\subsection{Additives and Test Oils Used}

The sulfurized olefins used in this study are shown in Table 1. These sulfurized olefins were commercial products. They were used as received or after fractionation by silica gel chromatography (solvent extraction method, [14]). The sulfur chain lengths and structures of the alkyl groups in the sulfurized olefins were determined using C-13 nuclear magnetic resonance (NMR) and field desorption mass spectrometry (FD-MS). The sulfurized olefins with names ending in " $\mathrm{A}$ " contained sulfur chains in which the average number of sulfurs was at least three, and those with names ending in " $\mathrm{D}$ " contained an average of three sulfur chains. Generally, when referring to sulfur-based EP-additives, the former " $\mathrm{A}$ " type is categorized as active while the latter " $\mathrm{D}$ " type is categorized as inactive. Figure 1 shows the sulfur chain lengths found in the sulfurized olefins and their abundance ratio. Both iC $8 \mathrm{~A}$ and $\mathrm{iC} 8 \mathrm{D}$ were produced from a dimerized isobutene feedstock. The sulfur chain length distribution ranged from 3 to 8 for iC $8 \mathrm{~A}$, whereas the sulfur chain length of iC8D was only three. nC12A was produced from a 1-Dodecene feedstock. We confirmed that three types of compounds (A, B, and C in Table 1) were produced in the manufacturing process. The sulfur chain length distribution ranged from 2 to 7 . iC8A and $\mathrm{nC} 12 \mathrm{~A}$ were fractionated to make their sulfur chain distributions as similar as possible (Figure 1).

The overbased calcium compounds used in this study are shown in Table 2. We used a commercial, synthetic calcium sulfonate (Base Number: $400 \mathrm{mg} \mathrm{KOH} / \mathrm{g}$, calcium content: 15 mass \%, abbreviated as $\mathrm{Ca}-\mathrm{Su}$ ). Ca-Su was produced from a soft alkyl benzene feedstock. The alkyl chain length was analyzed by negative ion fast atom bombardment mass spectrometry. The calcium salicylate (Base Number: $320 \mathrm{mg} \mathrm{KOH} / \mathrm{g}$, calcium content: 12 mass \%, abbreviated as Ca-Sa) used in this study was also a commercial product. 
The test oils were prepared by adding each sulfurized olefin to mineral base oil such that the total sulfur content was 10 mass \%. The test oils were further prepared by combining the sulfurized olefin iC8A with Ca-Su or Ca-Sa so that the total calcium content was 5 mass \%. The oil prepared with a combination of iC8A and Ca-Su was designated "iC8A + Ca-Su". The oil prepared with iC8A and Ca-Sa was designated "iC8A + Ca-Sa". In order to eliminate the influence of the viscosity, the kinematic viscosity of the test oils was adjusted to $100 \mathrm{~mm}^{2} / \mathrm{s}$ at $40{ }^{\circ} \mathrm{C}$.

Table 1. Sulfurized olefin samples.

\begin{tabular}{cccc}
\hline Sample name & $\begin{array}{c}\text { Sulfur chain length: } \\
\text { Molecular structure: }\end{array}$ & $\begin{array}{c}\text { Alkyl: } \\
\mathbf{R}\end{array}$ \\
\hline C8 (Branched) & C8 (Branched) \\
3-8 (Active) & 2-7 (Active) & C12 (Straight) \\
\end{tabular}

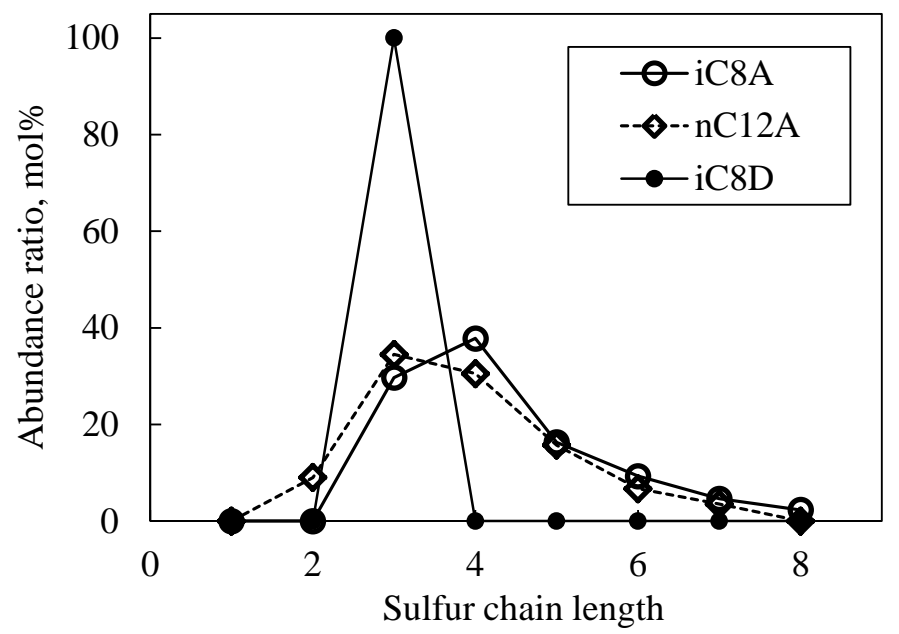

Figure 1. Sulfur chain lengths in the sulfurized olefins used.

Table 2. Overbased calcium compound samples.

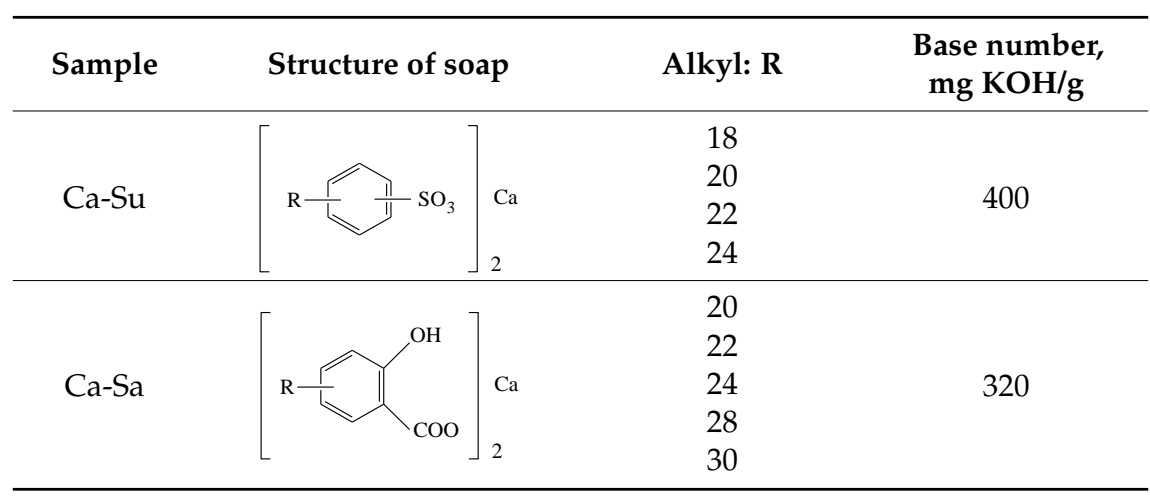




\subsection{Burring Test}

\subsubsection{Test Procedure}

The test oils were evaluated using a burring test which was devised and reported by Kitamura et al. [12] and Harada [13]. They reported that the burring test simulated the conditions of the ironing process and could be used to evaluate the anti-galling performance of lubricating oils. A schematic outline of the burring test is shown in Figure 2. For this test, the workpiece was austenitice stainless steel (JIS SUS304, Nisshin Steel Co., Ltd., Tokyo, Japan) and we used a ball made of high carbon-chromium bearing steel (JIS SUJ2, TSUBAKI NAKASHIMA Co., Ltd., Katsuragi, Japan). Using a dropper, $0.5 \mathrm{~g}$ of the test oil was applied to the central hole of the workpiece. The ball was then placed on the central hole. With a blank-holding force applied to the workpiece, the punch pushed the ball down. We used a model TM080F press machine manufactured by the Amino Corporation (Fujinomiya, Japan). The conditions of the burring test are shown in Table 3.

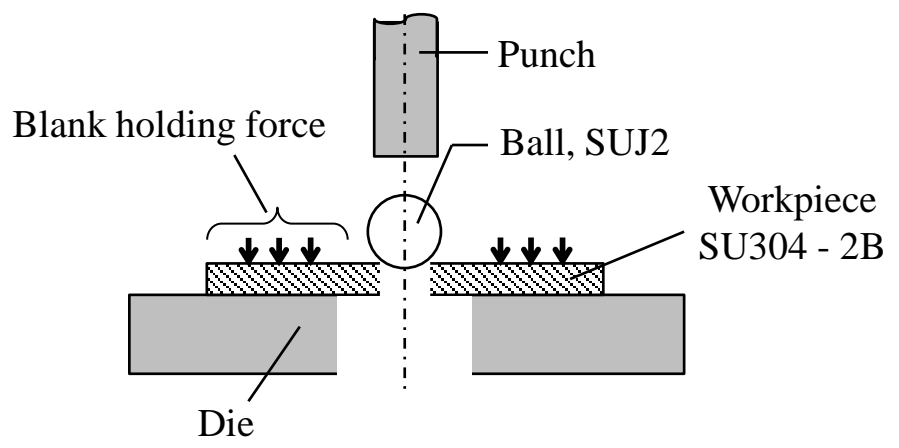

Figure 2. Principle of the burring test.

Table 3. Experimental conditions of the burring test.

\begin{tabular}{ccc}
\hline \multirow{3}{*}{ Workpiece } & Material & SUS304-2B (in JIS *) \\
& Diameter, mm & 80 \\
& Thickness, mm & 2 \\
& Hole diameter, mm & 4 \\
\hline \multirow{2}{*}{ Ball } & Quality of material & SUJ2 (in JIS *) \\
& Diameter, mm & 14.25 \\
\hline \multicolumn{2}{c}{ Test speed, mm/s } & 10 \\
\multicolumn{2}{c}{ Blank holding force, kN } & 19.6 \\
\hline \multicolumn{2}{c}{ * JIS: Japanese Industrial Standards. }
\end{tabular}

\subsubsection{Evaluation Method of the Ironing Performance}

The burring test involves two processes: bending and ironing. The relationship between punch stroke and penetration load is shown in Figure 3. In the ironing process, the difference in ironing performance between the good and poor test oils becomes apparent. Oils that perform poorly will show a trend similar to the broken line of the "Poor oil" curve in Figure 3. This is because the ball surface has been galled or otherwise damaged as shown in Figure 4. Ironing performance was evaluated based on the energy required to push the ball through the workpiece (Penetration Energy, abbreviated as P.E.). The P.E. is calculated by integrating the record of the relationship between the punch stroke and penetration load. The lower the value of P.E., the higher the performance of the test oil. The test was conducted at least five times for each oil and the values were averaged after discarding the maximum and minimum values. This average value was taken as the test result. 


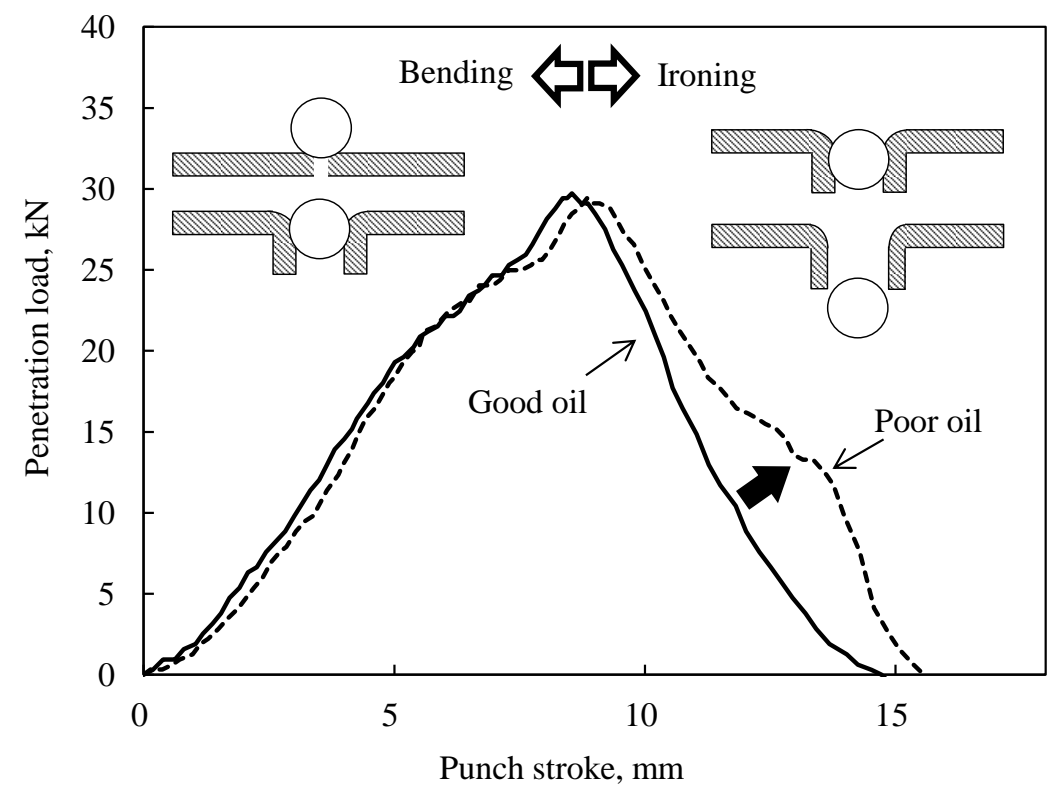

Figure 3. Example of relationship between punch stroke and penetration load.

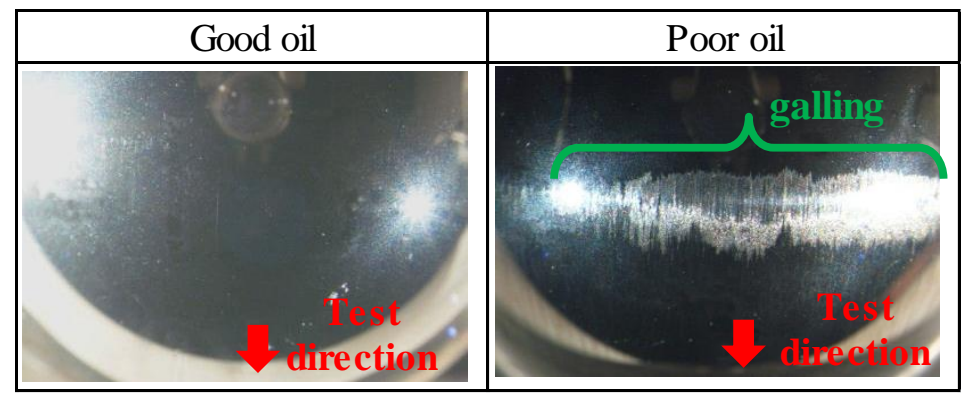

Figure 4. Example of the surface appearance of the ball after the burring test.

\subsection{Surface Analysis}

After the burring test with the test oils, lubricating films on the workpieces were analyzed by X-ray photoelectron spectroscopy (XPS) and Auger electron spectroscopy (AES). The workpieces were dry-cut to obtain test pieces, which were subjected to ultrasonic cleaning with n-hexane and were then analyzed by XPS and AES.

XPS was used to determine the chemical structure of the tribofilm. To determine the thickness of the tribofilm, AES depth profiles were obtained by argon ion sputtering. Although film thickness is not typically determined by AES, for our purposes, film thickness was taken to be the depth at which the oxygen concentration was $50 \%$ of the maximum value [15]. The conditions of these surface analyses are shown in Table 4. The point of analysis was midway along the sliding surface (A) of the workpiece, as shown in Figure 5. Furthermore, to determine the chemical structure of the tribofilms of $\mathrm{iC} 8 \mathrm{~A}, \mathrm{iC} 8 \mathrm{~A}+\mathrm{Ca}-\mathrm{Su}$, and iC8A + Ca-Sa in greater detail, the take-off angle of the photoelectrons in XPS was varied from $75^{\circ}$ to $15^{\circ}$. The standard take-off angle was $45^{\circ}$, which gave a sampling depth of about $4 \mathrm{~nm}$. With the angle-resolved XPS, the sampling depth was about $5 \mathrm{~nm}$ with a take-off angle of $75^{\circ}$, and about $1 \mathrm{~nm}$ with a take-off angle of $15^{\circ}$. 
Table 4. XPS and AES analysis conditions.

\begin{tabular}{lll}
\hline \multirow{4}{*}{ XPS } & Device name: & Quantum-2000 (manufactured by PHI) \\
\cline { 2 - 3 } & X-ray source: & Monochromatic-Al-K $\alpha$ radiation $(1486.6 \mathrm{eV})$ \\
\cline { 2 - 3 } & Analysis area: & DIA $200 \mu \mathrm{m}$ (elliptical shape) \\
\cline { 2 - 3 } & Take-off angle of the photoelectrons: & $\frac{45^{\circ}(\text { standard })}{15^{\circ}, 75^{\circ} \text { (angle-resolved XPS) }}$ \\
\hline \multirow{2}{*}{ AES } & Device name: & Model-680 (manufactured by PHI) \\
\cline { 2 - 3 } & Electron accelerating voltage: & $10 \mathrm{kV}$ \\
\cline { 2 - 3 } & Ion accelerating voltage: & $3 \mathrm{kV}$ \\
\cline { 2 - 3 } & Sputtering rate: & $18 \mathrm{~nm} / \mathrm{min}$ (relative to SiO2) \\
\hline
\end{tabular}

Analyzing inner surface

where ball penetrated

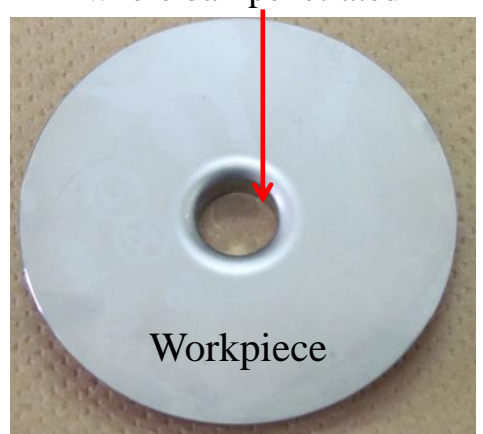

Enlarged view of inner surface

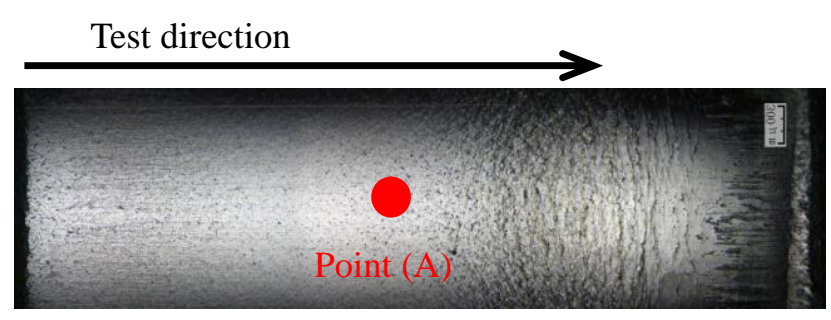

Figure 5. The analysis point of XPS and AES.

\section{Results and Discussion}

\subsection{Results of the Burring Test}

The burring test results for the sulfurized olefins alone are shown in Figure 6. Based on its lower P.E., iC8A performed better than iC8D. The sulfurized olefin which had longer sulfur chains showed higher ironing performance. It had been reported that sulfurized olefins with longer sulfur chain lengths typically show higher lubrication performance [9]. Our results were consistent with those of previous studies. Comparing samples iC8A and nC12A, the former slightly outperformed the latter. This differed from the results of a cutting test reported in a previous study [9]. In that study, straight chain types performed better than branched types. Sembongi et al. explained that this is because, typically, straight chain types adsorb more densely to metal surfaces than branched types, such that the adsorption film performs better. On the other hand, we speculate that the reason the branched type performed better in this study may be related to its thermal decomposition characteristics. Typically, organo-sulfur compounds are initially adsorbed on the iron surface and then cleavage of the sulfur-sulfur (S-S) bond occurs so that iron sulfide is formed. As loads approaching EP conditions are reached, the increasing temperature in the contact zone causes cleavage of the carbon-sulfur (C-S) bond as well as the S-S bond to occur, giving an inorganic sulfur-containing layer on the iron sulfide [16]. Looking closer at the molecular structure of the sulfurized olefins, we saw that the carbons bonded to sulfurs in nC12A are primary or secondary carbons, while those in iC8A are tertiary carbons. The carbon radical in the form of tertiary carbon is generally more stable than those of secondary carbon and primary carbon. If we assume that the bond dissociation energy for $\mathrm{C}-\mathrm{S}$ differs for $\mathrm{iC} 8 \mathrm{~A}$ and $\mathrm{nC} 12 \mathrm{~A}$, the $\mathrm{C}-\mathrm{S}$ bonds in $\mathrm{iC} 8 \mathrm{~A}$ might be more easily cleaved, so that iron sulfide would tend to form more easily with iC $8 \mathrm{~A}$. We therefore predicted that since the lubrication conditions in the burring 
test are more severe, the ironing performance would depend on the properties of the reaction film, which is related to the thermal decomposition characteristics, rather than the adsorption film.

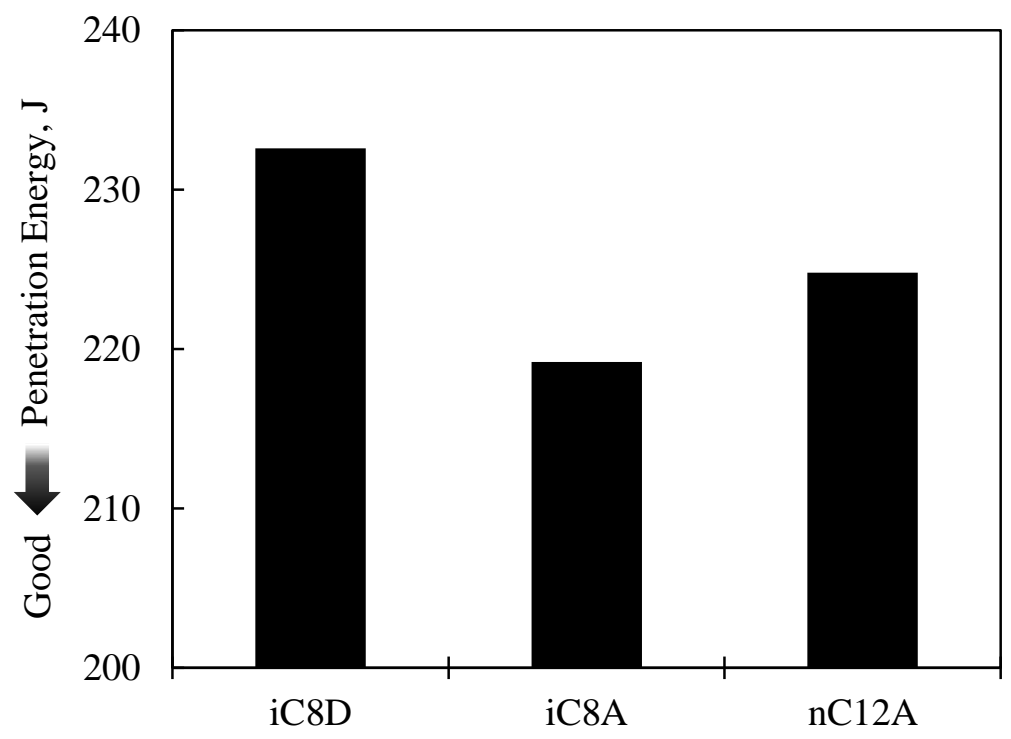

Figure 6. The penetration energy of single sulfurized olefins.

The burring test results for iC8A when used in combination with overbased calcium compounds are shown in Figure 7. By virtue of its lower P.E., iC8A + Ca-Su had a higher performance than iC8A. Meanwhile, iC8A + Ca-Sa also had a higher performance than iC8A. This test revealed the combined effect of sulfurized olefins and salicylate for the first time. However, the samples prepared with sulfonate and salicylate performed almost the same. Thus, the ironing performance was not affected by the difference in structure of the soap groups.

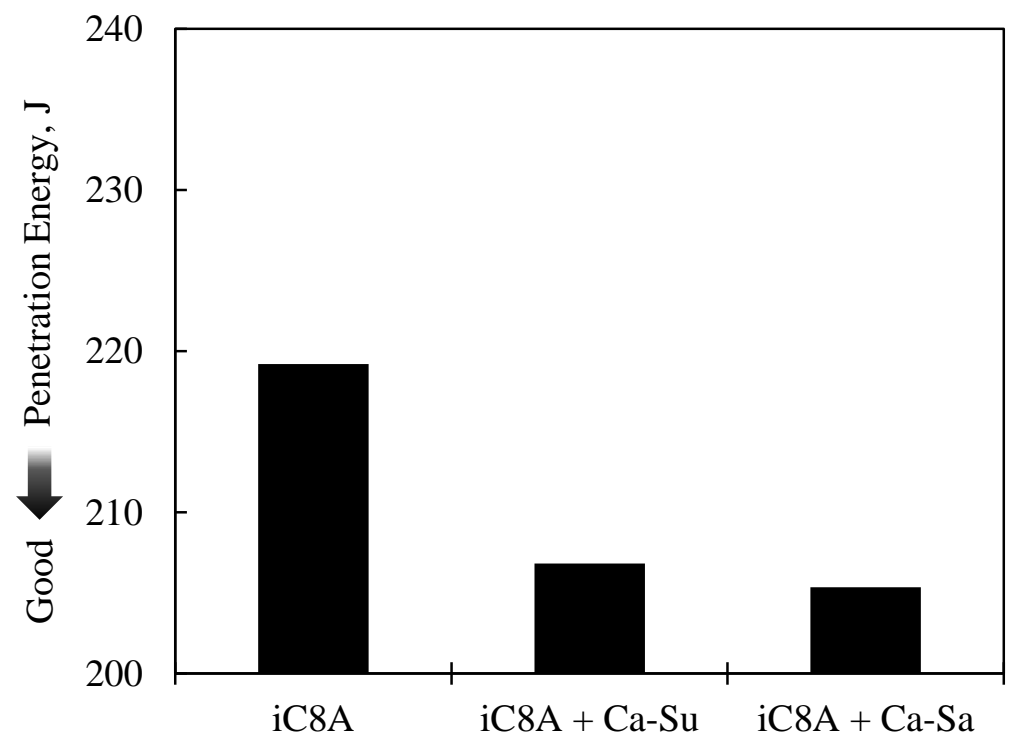

Figure 7. The penetration energy of the combination of sulfurized olefin and calcium compounds. 


\subsection{Results of Surface Anaylsis}

\subsubsection{Sulfurized Olefins Alone}

Using XPS and Auger AES, we analyzed the surface of the workpiece for samples iC8A and $\mathrm{nC12} \mathrm{A}$ after the burring test. The ratio of sulfur to iron $(\mathrm{S} / \mathrm{Fe})$ as determined by XPS is shown in Figure 8. Meanwhile, Figure 9 shows the binding energy of sulfur. As Figure 8 shows, iC8A had a higher S/Fe ratio than nC12A. As Figure 9 shows, peaks were detected at around 162 and $168 \mathrm{eV}$ for both iC8A and nC12A. The former is attributed to sulfide, and the latter is attributed to sulfate, sulfite, and sulfonate. The peak at around $162 \mathrm{eV}$ is thought to be iron sulfide which was formed in a reaction between the iron surface and sulfurized olefins. Meanwhile, the peak at around $168 \mathrm{eV}$ is attributed to iron sulfate and/or sulfite. This iron sulfide may be oxidized by the heat generated by the ironing process. According to reference [16], our guess is that the iron sulfide had changed to iron sulfate and/or sulfite, as illustrated in Figure 10. The speculation that the iron sulfide was oxidized is also supported by the analysis results which follow. To determine the composition of the tribofilm for iC8A in more detail, we conducted angle-resolved XPS. Figure 11 shows the binding energy of sulfur at various depths. It was found that the peak intensity around $168 \mathrm{eV}$ increased closer to the outermost surface. This meant that the proportion of iron sulfate and iron sulfite in the tribofilm was higher at the positions closer to the outermost surface. This indicated that the temperature on the outermost surface side was higher, and that the film of the outermost surface side was more likely to be oxidized.

Figure 12 shows the tribofilm thickness as measured by AES. The tribofilms with both iC8A and nC12A were almost the same in thickness.

Our results showed that the lubricating films with both iC8A and nC12A were almost identical in chemical species and thickness. However, the S/Fe ratio of iC8A was higher than that of nC12A. It is thought that a denser layer of iron sulfide formed with iC8A. The reason why iC8A showed superior ironing performance to $\mathrm{nC} 12 \mathrm{~A}$ seems to be related to the difference in the way that the iron sulfide reaction film forms with each compound.

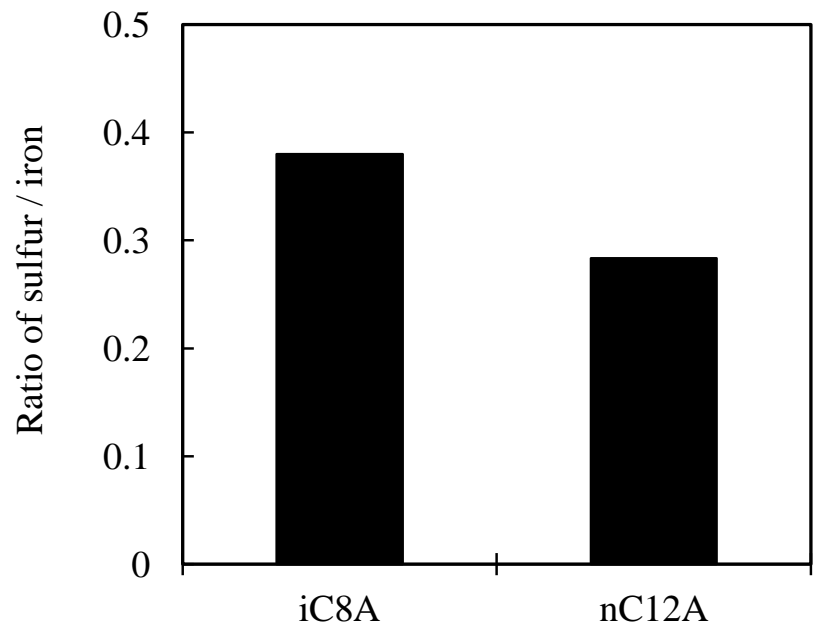

Figure 8. The ratio of sulfur to iron determined by XPS (Samples: iC8A and nC12A). 


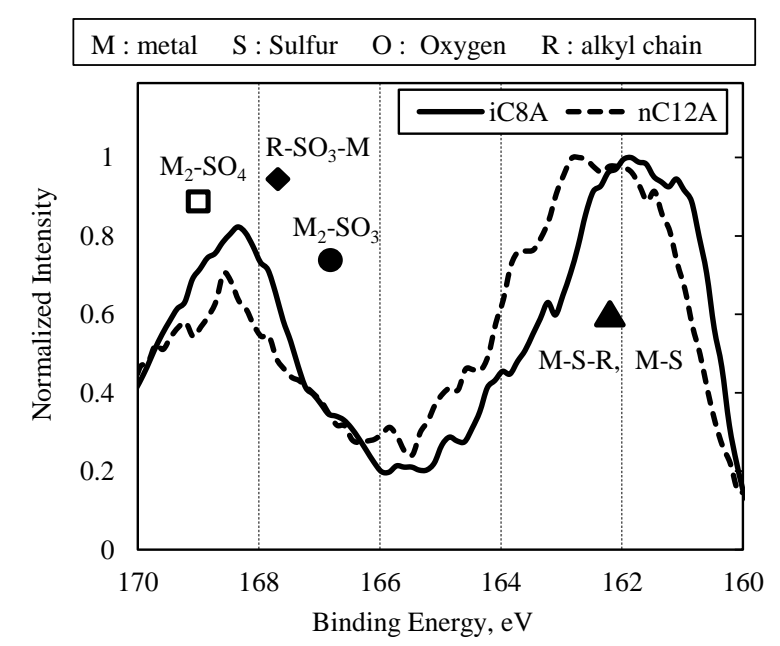

Figure 9. XPS spectra: S2p (Samples: iC8A and nC12A).

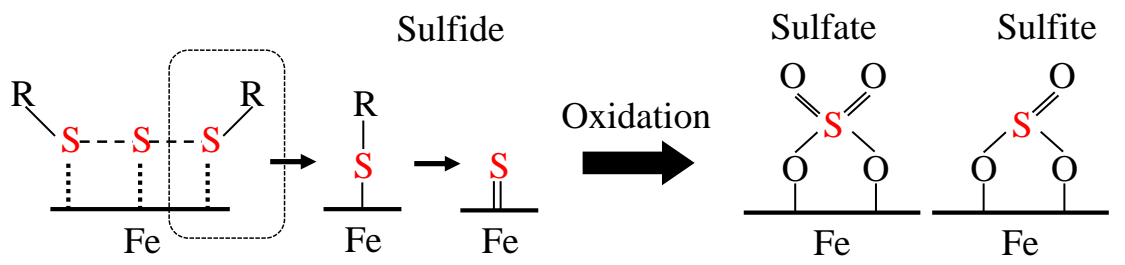

Figure 10. Image figure of reaction of iron sulfide being oxidized.

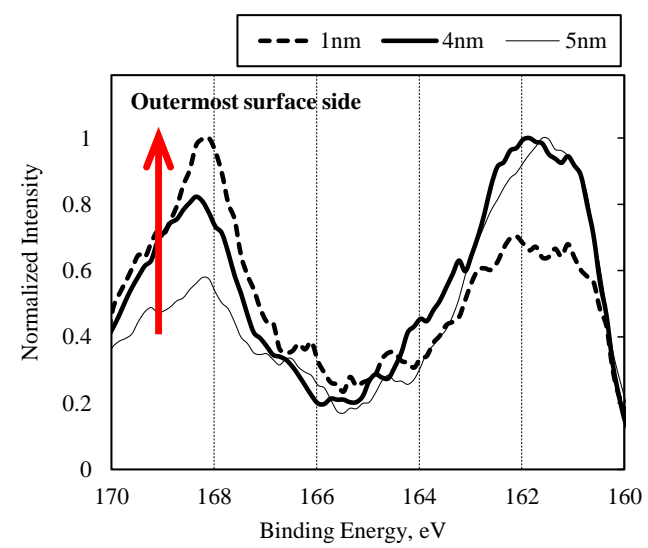

Figure 11. Angle resolved XPS spectra: S2p (Sample: iC8A).

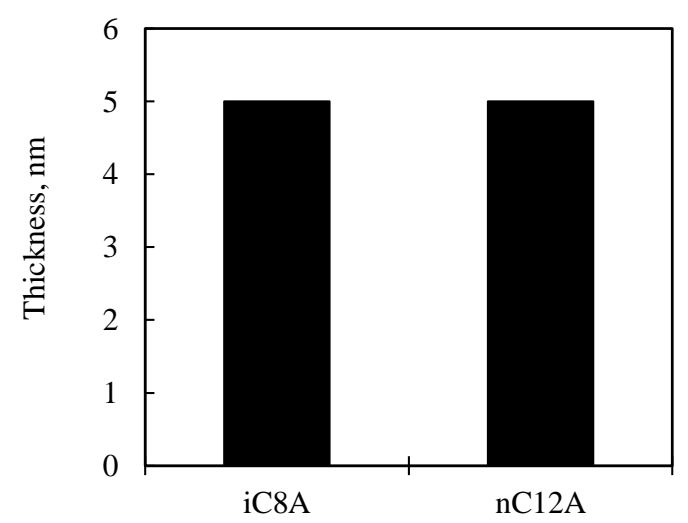

Figure 12. Tribofilm thickness measured by AES (Samples: iC8A and nC12A). 


\subsubsection{Combination of Sulfurized Olefins with Overbased Calcium Compounds}

Figure 13 shows the ratio of sulfur to iron $(\mathrm{S} / \mathrm{Fe})$ as measured by XPS. The ratio of calcium to iron $(\mathrm{Ca} / \mathrm{Fe})$ is shown in Figure 14. Figures 15-17 show the binding energy of each element (sulfur, calcium, or carbon). Figures 18 and 19 show the binding energy of sulfur and calcium at various depths by angle resolved XPS. Figure 20 shows the tribofilm thickness as measured by AES.

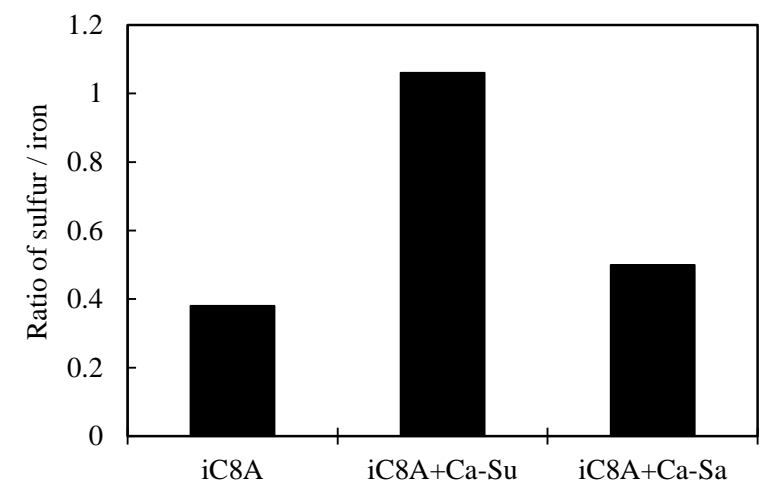

Figure 13. Ratio of sulfur to iron measured by XPS (Samples: iC8A, iC8A + Ca-Su, and iC8A + Ca-Sa).

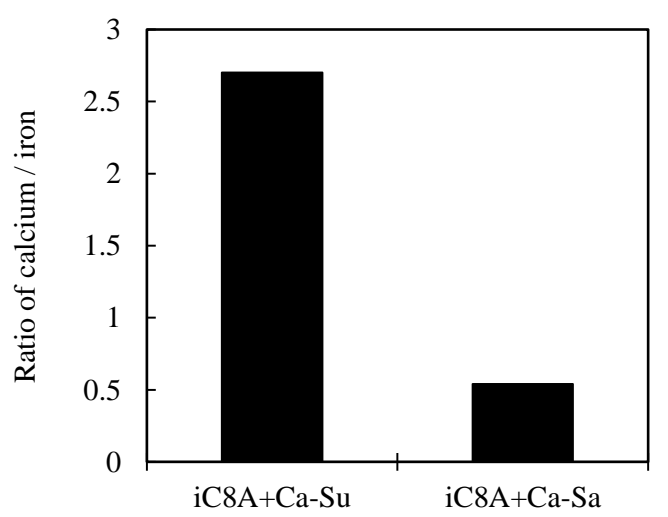

Figure 14. Ratio of calcium to iron measured by XPS (Samples: iC8A, iC8A + Ca-Su, and iC8A + Ca-Sa).

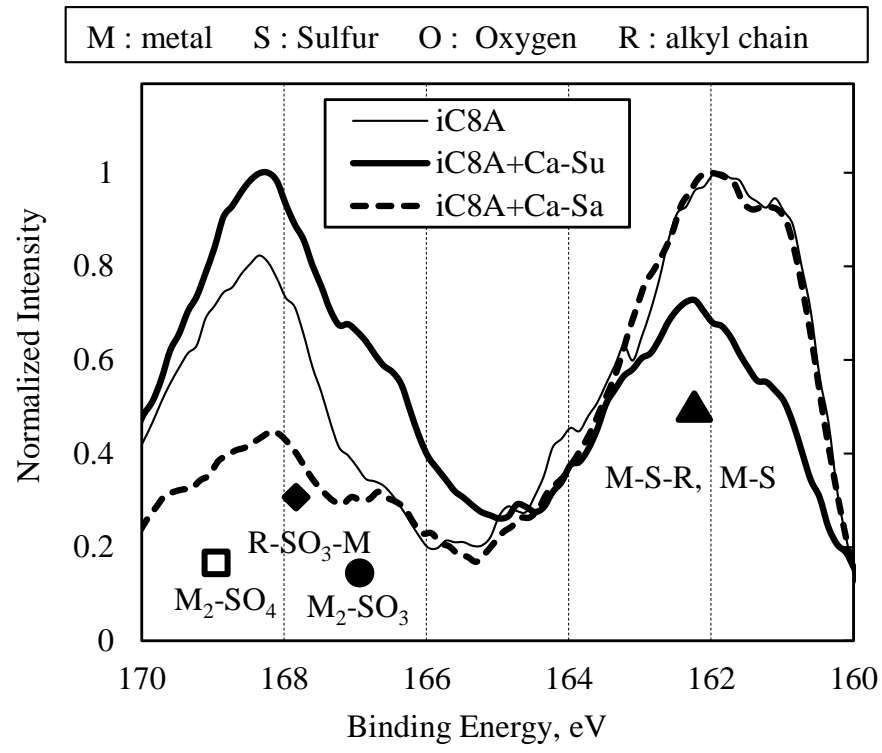

Figure 15. XPS spectra: S2p (Samples: iC8A, C8A + Ca-Su, and iC8A + Ca-Sa). 


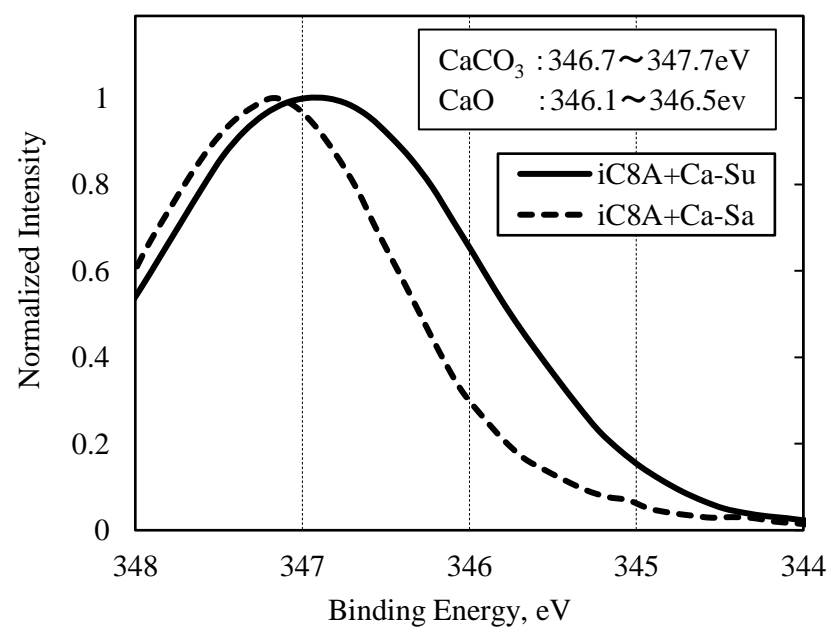

Figure 16. XPS spectra: Ca2p (Samples: iC8A + Ca-Su and iC8A + Ca-Sa).

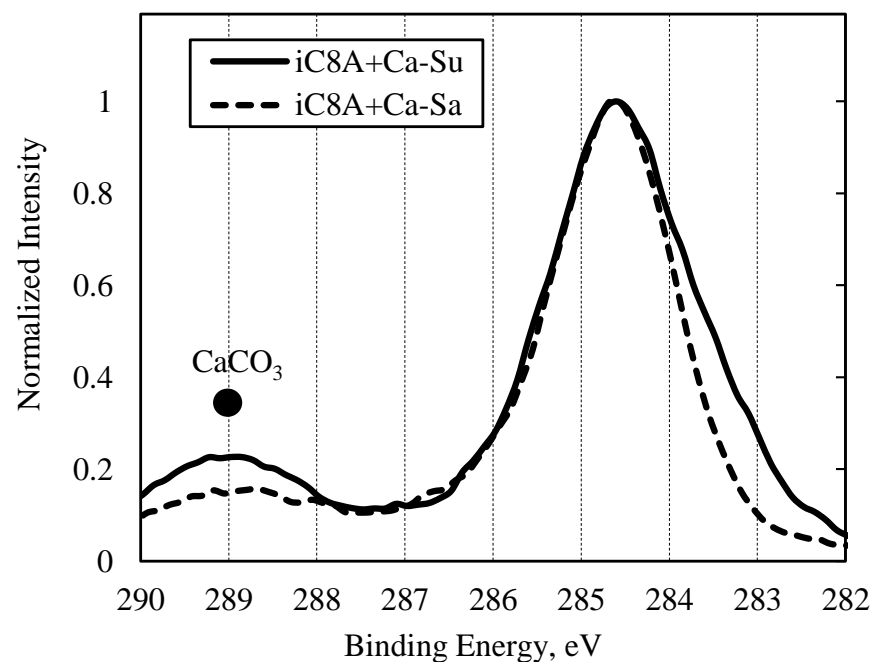

Figure 17. XPS spectra: C1s (Samples: iC8A + Ca-Su and iC8A + Ca-Sa).

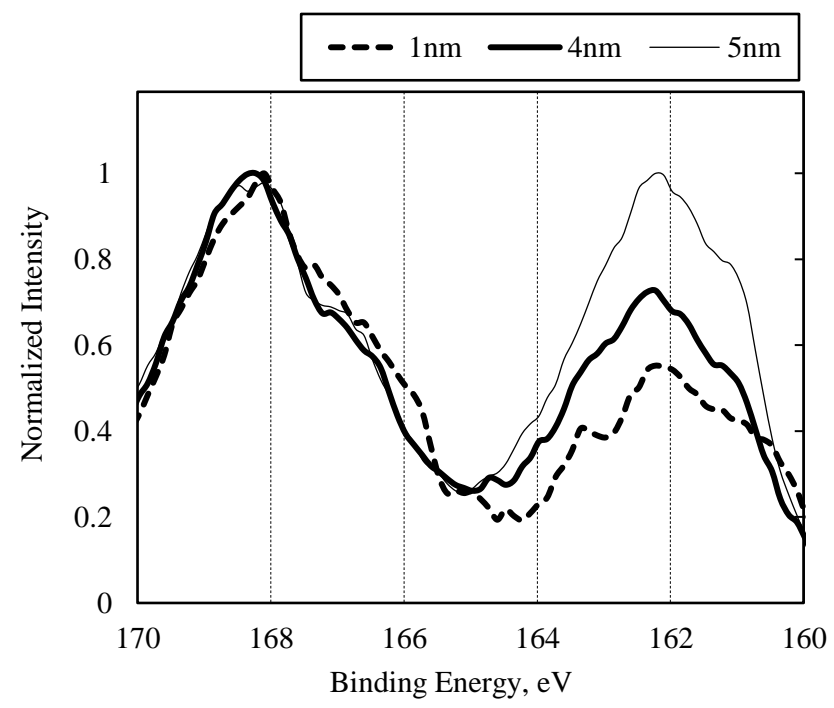

(a)

Figure 18. Cont. 


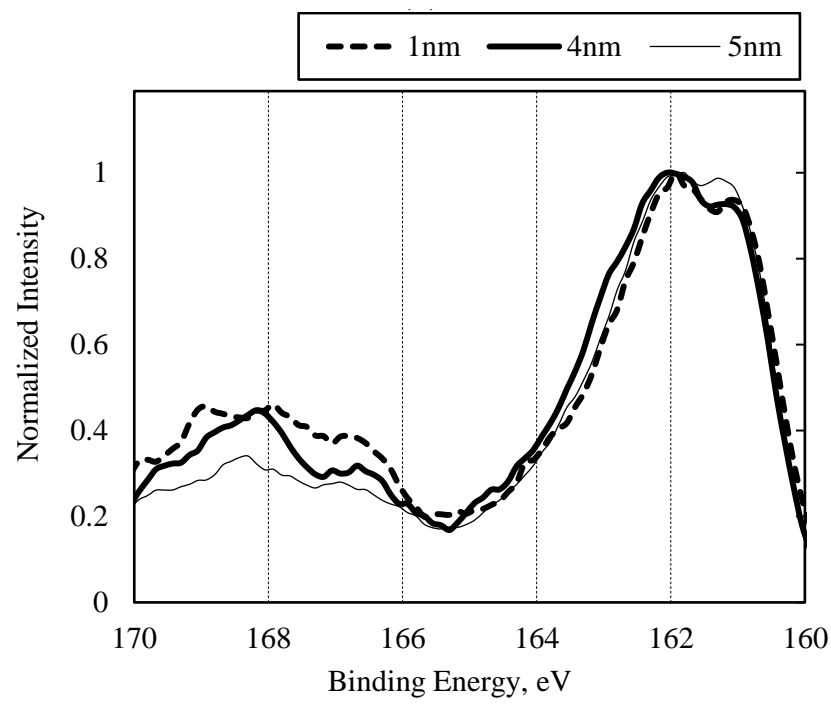

(b)

Figure 18. (a) Angle resolved XPS spectra: S2p (Sample: iC8A + Ca-Su); (b) Angle resolved XPS spectra: S2p (Sample: iC8A + Ca-Sa).
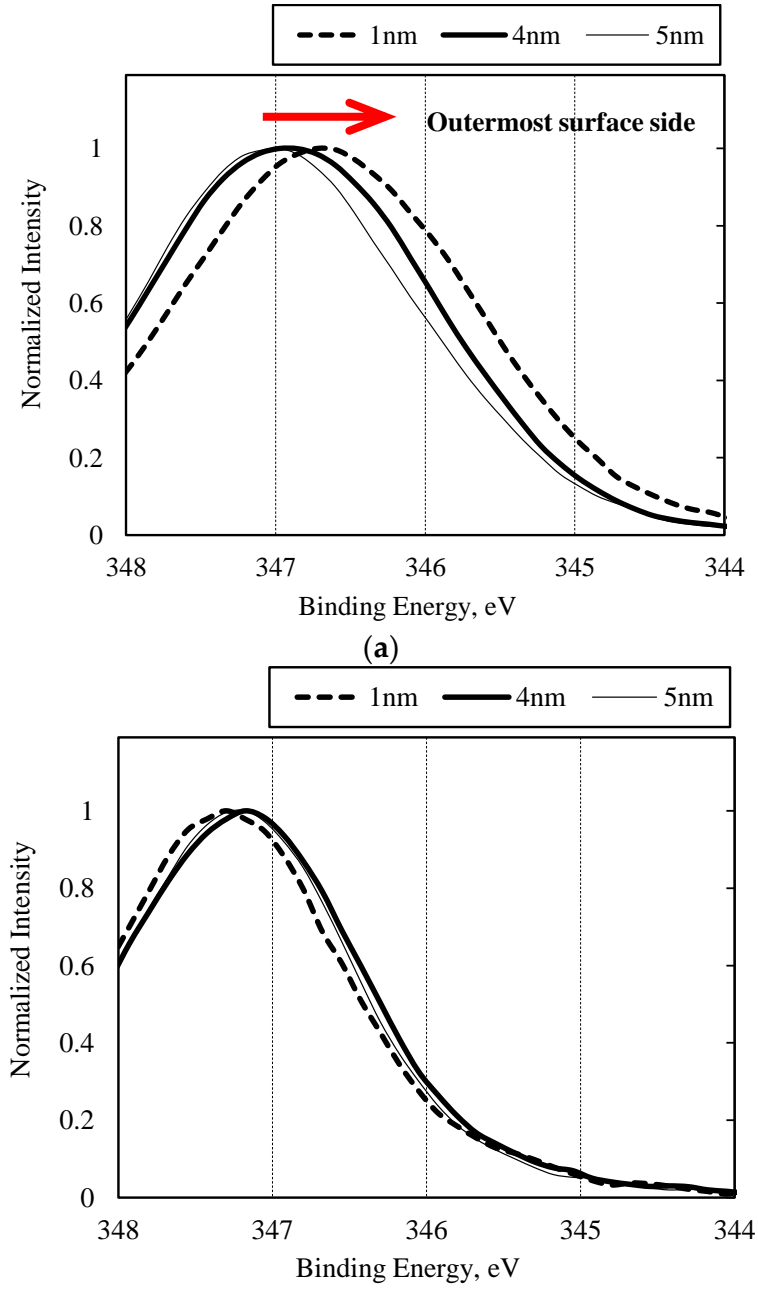

(b)

Figure 19. (a) Angle resolved XPS spectra: Ca2p (Sample: iC8A + Ca-Su); (b) Angle resolved XPS spectra: Ca2p (Sample: iC8A + Ca-Sa). 


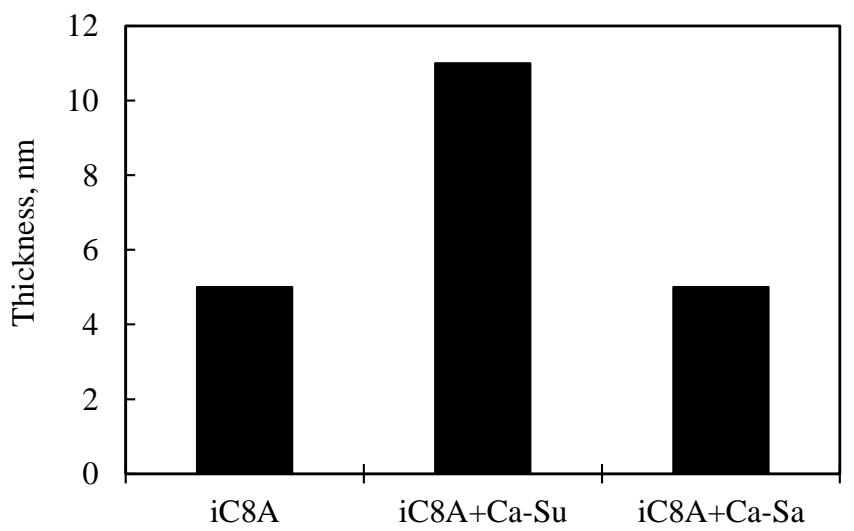

Figure 20. Tribofilm thickness measured by AES (Samples: iC8A, iC8A + Ca-Su, and iC8A + Ca-Sa).

In Figure 13, comparing samples iC8A and iC8A + Ca-Su, the S/Fe ratio of iC8A + Ca-Su was higher than that of iC8A. In Figure 15a, as with iC8A alone, peaks were detected at around $162 \mathrm{eV}$ and $168 \mathrm{eV}$ for iC8A + Ca-Su. However, their relative intensities were different and the highest peak was around $168 \mathrm{eV}$ for $\mathrm{iC} 8 \mathrm{~A}+\mathrm{Ca}-\mathrm{Su}$. Based on the chemical structure of the soaps (Table 2), our theory is that the soap was adsorbing on the metal surface, and that the strong peak around $168 \mathrm{eV}$ was associated with the adsorption film. As Figure 18a shows, the deeper the position in the film, the higher the relative intensity derived from sulfide. This suggests that the soap is being adsorbed on the outermost surface side as reported in the previous studies [6,17]. Calcium was abundantly detected on the surface with iC8A + Ca-Su (Figure 14). The binding energies of calcium (Ca2p) and carbon (C1s), shown in Figures 16 and 17, indicated that the chemical calcium species was calcium carbonate and/or calcium oxide. In Figure 19a, the chemical shift of the peak toward the lower energy side suggested that more calcium oxide was present on the outermost surface side. Calcium oxide is not originally contained in overbased calcium sulfonate, so our thought was that the calcium oxide had been formed by a tribochemical reaction $\left(\mathrm{CaCO}_{3} \rightarrow \mathrm{CaO}+\mathrm{CO}_{2}\right)$. In fact, such cases have been reported previously [6].

As Figure 13 shows, the S/Fe ratios with iC8A and iC8A + Ca-Sa were almost the same. This was due to the absence of sulfur in the soap structure of calcium salicylate. In Figure 15b, the strongest peak was detected at around $162 \mathrm{eV}$, and was attributed to iron sulfide. The peak intensity around $168 \mathrm{eV}$ was much lower for iC8A + Ca-Sa. In fact, this oil showed excellent ironing performance (Figure 7). We therefore speculated that the lower amount of heat generated by the process meant that the iron sulfide was less likely to oxidize. Calcium was also detected on the surface with iC8A + Ca-Sa, though the amount was small compared with iC8A + Ca-Su (Figure 14). The chemical calcium species was found to be calcium carbonate and/or calcium oxide (Figures 16 and 17). However, there was a difference in the peak widths with iC8A + Ca-Su and iC8A Ca-Sa (Figure 16). The wider peak width for $\mathrm{iC} 8 \mathrm{~A}+\mathrm{Ca}-\mathrm{Su}$ suggested that a greater proportion of calcium oxide had formed on the surface. Furthermore, there was a large difference in tribofilm thickness with iC8A + Ca-Su and iC8A + Ca-Sa (Figure 20). The tribofilm generated with iC8A + Ca-Su was thicker than that with iC8A. In contrast, iC8A and iC8A + Ca-Sa formed tribofilms of similar thickness. These differences between iC8A + $\mathrm{Ca}-\mathrm{Su}$ and iC8A + Ca-Sa will be the subject of future research.

Our results showed that iron sulfide and calcium carbonate were detected on the workpiece surface after the burring test with both iC8A + Ca-Su and iC8A + Ca-Sa. This suggests that lubricating films consisting of both iron sulfide and calcium carbonate should help to improve ironing performance.

\section{Conclusions}

In this study, we prepared specific organo-sulfur compounds with well-defined molecular structures and evaluated their ironing performance by way of the burring test, which simulates 
the conditions of cold ironing. Furthermore, we also evaluated the performance of these compounds when used in combination with calcium sulfonate or calcium salicylate. Based on the burring test results and surface analysis after the test, the following findings were obtained:

(1) With sulfurized olefins, sulfur chain length and alkyl structure play an important role in ironing performance. The branched type sulfurized olefins had better performance because a denser layer of iron sulfide was formed on the workpiece surface.

(2) When the sulfurized olefins were combined with overbased calcium sulfonate or salicylate, the performance was better than with sulfurized olefins alone. The combined effect of sulfurized olefins and salicylate was revealed for the first time.

(3) There were differences in the tribofilms formed with the oils prepared with calcium sulfonate and salicylate. However, what was common to both was that iron sulfide and calcium carbonate were detected on the surface of the workpiece after the burring test. Thus, lubricating films consisting of both iron sulfide and calcium carbonate seem to improve ironing performance.

Acknowledgments: The authors gratefully acknowledge the financial support from JX Nippon Oil \& Energy Corporation. We would also like to express sincere thanks to, Director, Komiya for encouragement and support in carrying out this work and granting permission to submit paper for publication in the journal "Lubricants".

Author Contributions: Tomohiro Takaki, Kazuhiro Yagishita and Teppei Tsujimoto conceived and designed the experiments; Tomohiro Takaki conducted experiments and wrote the paper; Toshiaki Wakabayashi introduced useful information and discussed for the article.

Conflicts of Interest: The authors declare no conflict of interest.

\section{References}

1. Hosford, W.F.; Caddell, R.M. Metal Forming Mechanics and Metallurgy, 4th ed.; Cambridge University Press: New York, NY, USA, 2011; pp. 106-112.

2. Allum, K.G.; Forbers, E.S. The Load-Carrying Mechanism of Organic Sulfur Compounds-Application of Electron Probe Microanalysis. ASLE Trans. 1968, 11, 162-175. [CrossRef]

3. Mori, S.; Hori, K.; Tamai, Y. Mechanochemical Reaction of Extreme Pressure Additive with Mild Steel. J. Jpn. Soc. Tribol. 1982, 27, 505-511. (In Japanese)

4. Kubo, T.; Minami, I.; Mori, S. Investigation of tribocemical reaction by organic sulfides on nascent metal surfaces. Tribol. Online 2007, 2, 89-92. [CrossRef]

5. Wakabayashi, T.; Yokota, H.; Okajima, M.; Ogura, S. Effect of Cutting Oils and Infuleence of Tool Materials on Metal Cutting (Part 1). J. Jpn. Soc. Tribol. 1994, 39, 784-791. (In Japanese)

6. Kubo, T.; Nanao, H.; Minami, I.; Mori, S. TOF-SIMS Analysis of Boundary Film Dervied from Multi-Additives. J. Jpn. Soc. Tribol. 2006, 51, 819-825. (In Japanese)

7. Kubo, T.; Nanao, H.; Minami, I.; Mori, S. TOF-SIMS Analysis of Boundary Film Dervied from Multi-Additives (Part 2)—Chemical Analysis with Depth Profiling. J. Jpn. Soc. Tribol. 2007, 52, 871-879. (In Japanese)

8. Costello, M.T.; Kasrai, M. Study of surface films of overbased sulfonates and sulfurized olefins by X-Ray Absorption Near Edge Structure (XANES) spectroscopy. Tribol. Lett. 2006, 24, 163-169. [CrossRef]

9. Sembongi, N.; Tsujimoto, T.; Takaki, T.; Yagishita, K.; Wakabayashi, T. Relationship between molecular structure of organo-sulfur compounds and machining performance. In Proceedings of the 19th International Colloquium Tribology, Stuttgard, Germany, 21-23 January 2014.

10. Takaki, T.; Ito, S.; Kitamura, K.; Yagishita, K.; Shibata, J. Relationship between Molecular Structures of Organic-Sulfur Compounds and Metal Forming Performance. Key Eng. Mater. 2016, 716, 190-198. [CrossRef]

11. Ohmori, T.; Kitamura, K.; Danno, A.; Kawamura, M. A Cold Forging Oil Containing Phosphorus Type EP Additives. Tribol. Trans. 1991, 34, 458-464. [CrossRef]

12. Mizuno, K.; Kitamura, K.; Sasai, A. An Experiment of Burring (Hole Flanging of Metal Sheet). In Proceedings of the 50th Japanese Joint Conference for the Technology of Plasticity, Fukuoka, Japan, 6-8 October 1999. (In Japanese)

13. Harada, T. Practical application of chlorine-free oils to press forming. Press Form. J. 2009, 8, 15-16. (In Japanese) 
14. Fujita, M. Component Analysis of Lubricants. J. Jpn. Soc. Tribol. 1967, 12, 5-14.

15. Yamamura, Y.; Shimizu, R.; Shimizu, H.; Itoh, N. Ion-Induced Sputtering. Shinku 1983, 26, 69-87. (In Japanese) [CrossRef]

16. Forbes, E.S.; Reid, A.J.D. Liquid Phase Adsorption/Reaction Studies of Organo-Sulfur Compounds and Their Load-Carrying Mechanism. ASLE Trans. 1973, 16, 50-60. [CrossRef]

17. Cizairea, L.; Martina, J.M.; Gresserb, E.; Dinhc, N.T.; Heau, C. Chemical analysis of overbased calcium sulfonate detergents by coupling XPS, ToF-SIMS, XANES, and EFTEM. Tribol. Lett. 2004, 238, 151-158. [CrossRef] article distributed under the terms and conditions of the Creative Commons Attribution (CC BY) license (http:/ / creativecommons.org/licenses/by/4.0/). 